\title{
Impact of the temperature ratio on turbulent impurity transport in Wendelstein 7-X
}

\author{
Th. Wegner, ${ }^{1, *}$ J. A. Alcusón, ${ }^{1}$ B. Geiger, ${ }^{1, \dagger}$ A. v. Stechow,${ }^{1}$ P. Xanthopoulos, ${ }^{1}$ C. Angioni, ${ }^{2}$ M. \\ Beurskens, ${ }^{1}$ L.-G. Böttger, ${ }^{1,}$ S. Bozhenkov, ${ }^{1}$ K. J. Brunner, ${ }^{1}$ R. Burhenn, ${ }^{1}$ B. Buttenschön, ${ }^{1}$ H. Damm, ${ }^{1}$ \\ E. Edlund, ${ }^{3}$ O. P. Ford, ${ }^{1}$ G. Fuchert, ${ }^{1}$ O. Grulke, ${ }^{1,}$ Z. Huang, ${ }^{4}$ J. Knauer, ${ }^{1}$ F. Kunkel, ${ }^{1}$ A. Langenberg,,${ }^{1}$ \\ N. A. Pablant, ${ }^{5}$ E. Pasch, ${ }^{1}$ K. Rahbarnia, ${ }^{1}$ J. Schilling, ${ }^{1}$ H. Thomsen, ${ }^{1}$ L. Vanó,${ }^{1}$ and W7-X Team ${ }^{1, \S}$ \\ ${ }^{1}$ Max-Planck Institute for Plasma Physics, 17491 Greifswald, Germany \\ ${ }^{2}$ Max-Planck Institute for Plasma Physics, 85748 Garching, Germany \\ ${ }^{3}$ SUNY Cortland, Cortland, NY 13045, USA \\ ${ }^{4}$ MIT Plasma Science and Fusion Center, Cambridge, MA 02139, USA \\ ${ }^{5}$ Princeton Plasma Physics Laboratory, Princeton, NJ 08543, USA
}

\begin{abstract}
First experimental observations in the Wendelstein 7-X stellarator indicate that the impurity confinement can be explained by turbulent processes. In particular, plasma discharges with increased ion to electron temperature ratio are accompanied by reduced electron density fluctuation amplitudes and anomalous impurity diffusion, suggesting a lower turbulent transport. Employing gyro-kinetic numerical simulations, we argue that the temperature ratio plays a key role for reducing the ion temperature gradient instability in Wendelstein 7-X, leading to an enhanced impurity confinement.
\end{abstract}

Keywords: impurity transport, turbulence, ion temperature gradient instability, stellarator, laser blow-off

\section{INTRODUCTION}

One of the most prominent challenges in magnetic fusion research is to improve and control the confinement properties of heat, particles and momentum by collisional and anomalous transport. Collisional transport is caused by Coulomb collisions and drifting orbits of charged particles, leading to so-called classical and neoclassical transport, which is subsumed in the following as "neoclassical transport". Any transport beyond the well understood neoclassical mechanisms is termed as anomalous and is generally attributed to turbulent fluctuations or magnetohydrodynamics modes. In stellarators, the neoclassical transport can be the dominant mechanism and causes non-ambipolar particle fluxes, due to orbit losses in the three-dimensional magnetic field geometry. The imposed separation of electrons and main plasma ions produces strong electric fields which are in turn linked to significant convective impurity fluxes. In case of high density operation with comparable electron and ion temperatures, negative electric fields develop such that heavy impurities tend to accumulate in the plasma core $[1,2]$. However, it is of utmost importance to avoid impurity accumulation since the associated radiative cooling can severely degrade the performance of a fusion device [3]. Possibly benign levels of turbulence can help avoiding impurity accumulation without a strong degradation of the overall plasma performance. However, turbulence is not well understood.

At the world's largest stellarator experiment Wendelstein 7$\mathrm{X}(\mathrm{W} 7-\mathrm{X})$, which is neoclassically optimized [4-7] and quasiisodynamic, an anomalous impurity transport was reported

\footnotetext{
* thomas.wegner@ipp.mpg.de,physics@thwegner.com

† Present address: University of Wisconsin-Madison, Madison, WI 53706, USA

$¥$ Also affiliated with the Technical University of Denmark, 2800 Kongens Lyngby,Denmark

$\S$ Author list in T. Klinger et al. Nucl. Fusion 59, 112004 (2019)
}

based on confinement studies of Argon ions [8]. Additionally, in recent investigations of core electron-root confinement plasmas (positive radial electric fields), the sum of the classical and neoclassical transport can only describe a small fraction of the impurity flux and an anomalous diffusion coefficient for the impurities is inferred, which is more than two orders of magnitude larger than the theoretical classical and neoclassical estimates [9].

In the present work, we address whether this observed level of anomalous transport in W7-X is caused by turbulence since, as any other stellarator to date, W7-X is not optimized for turbulent transport. Typically, three types of turbulent fluctuations at different scale lengths are considered in a fusion device which are the electron temperature gradient (ETG), the trapped electron mode (TEM) and the ion temperature gradient instability (ITG). Conducting GENE code [10] simulations, we are able to infer the dominant instabilities which contribute to the turbulent transport. ETG at electron scales is thought to play only a minor role in quasi-isodynamic stellarators [11] and should additionally not affect the impurity transport due to its small scale length. Both, the TEM and the ITG are characterized by similar scales in the order of the ion gyro-radius which is relevant for impurity transport.

Particularly for W7-X, TEM turbulence level is generally low $[12,13]$. Thus in W7-X, we expect mainly ITG modes [14-16] which are driven by the normalized ion temperature gradient length $a / L_{T_{\mathrm{i}}}=-\left(a / T_{\mathrm{i}}\right) \mathrm{d} T_{\mathrm{i}} / \mathrm{d} r(a=0.52 \mathrm{~m}$ is the minor radius of the torus and $r$ the effective plasma radius) at locations where the magnetic field curvature is locally unfavorable, and are stabilized by the normalized electron density gradient length $a / L_{n_{\mathrm{e}}}=-\left(a / n_{\mathrm{e}}\right) \mathrm{d} n_{\mathrm{e}} / \mathrm{d} r$. The ITG mode can only be excited if $a / L_{T_{\mathrm{i}}}$ is larger than a critical value, $a / L_{T_{\mathrm{i}, \text { crit }}}$. In many regimes of interest, $a / L_{T_{\mathrm{i}, \text { crit }}}$ increases with ion to electron ratio $\tau=T_{\mathrm{i}} / T_{\mathrm{e}}$, as it has been shown in axisymmetric geometry [15-17]. In W7-X, a similar increase of $a / L_{T_{\mathrm{i} \text {, rit }}}$ with $T_{\mathrm{i}} / T_{\mathrm{e}}$ has also been predicted [18]. It is thus expected that with an increasing $T_{\mathrm{i}} / T_{\mathrm{e}}$, the critical normalized ion temperature gradient length will also increase (almost lin- 


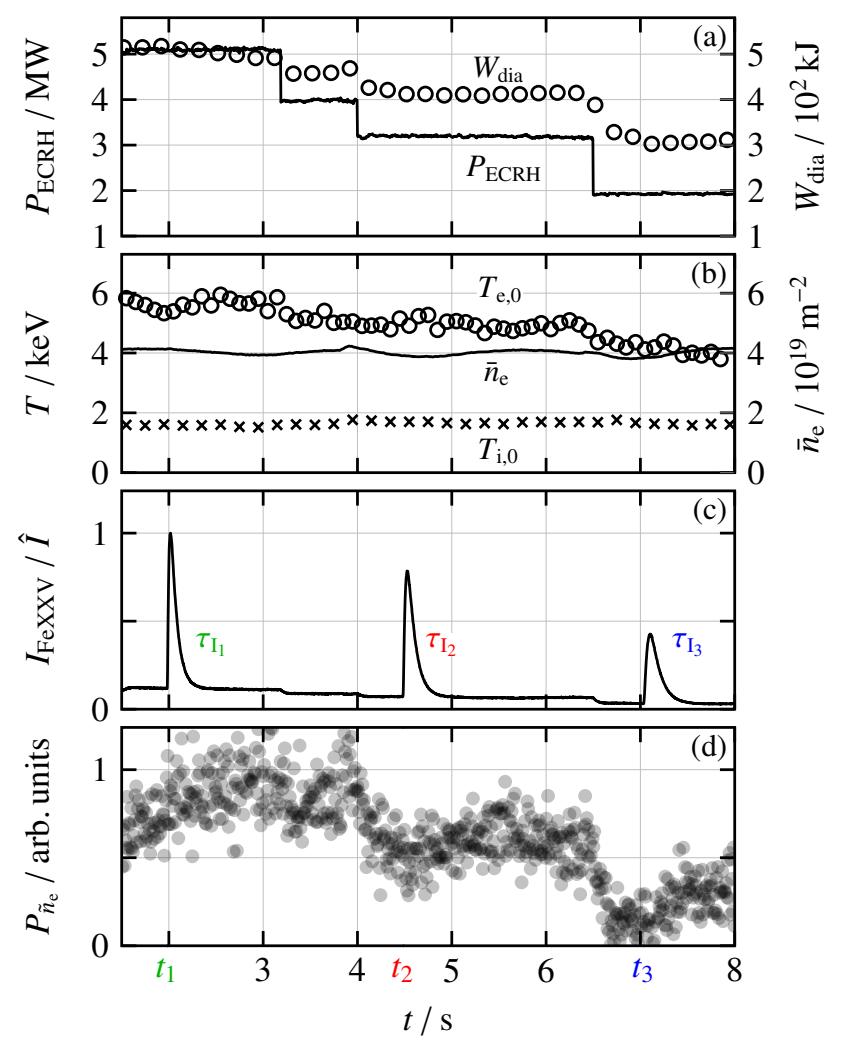

Figure 1. Time traces of the ECRH power (-) and the plasma stored energy (o) (a), the electron $(\circ)$ and ion $(x)$ temperature in the plasma center as well as the line-integrated electron density (-) (b), the normalized Fe XXV line emission (c) and the density fluctuation amplitude normalized to the line-averaged electron density (d) for the experimental program 20180906.038.

early for a flat density profile), and the ITG turbulence level will be reduced. These predicted dependencies on the temperature ratio are investigated in dedicated experiments. Hence, the competition between neoclassical and turbulent transport is examined and this Letter demonstrates a first evidence of turbulent dominated impurity transport in W7-X.

\section{EXPERIMENTAL SETUP}

To study the impact of the temperature ratio, experiments during the third operation phase (OP1.2b) of W7-X where performed. Hydrogen was used as fuel and the standard magnetic field configuration (labeled as EJM [19]) was employed. As illustrated in figure 1 (a), the on-axis (deposition within $0 \leq r / a \leq 0.15$ ) ECRH power was stepwise decreased from 5.2 to $2 \mathrm{MW}$. The electron density and temperature profiles were determined by a Thomson scattering diagnostics [20]. The absolute values of the density profile were cross-calibrated with the line-integrated electron density measured by a dispersion interferometer. The ion temperature profile was obtained by fitting the Doppler broadening of a carbon emission line $\left(\mathrm{C} \mathrm{VI}^{*}\right)$ measured by charge exchange recombination spec-
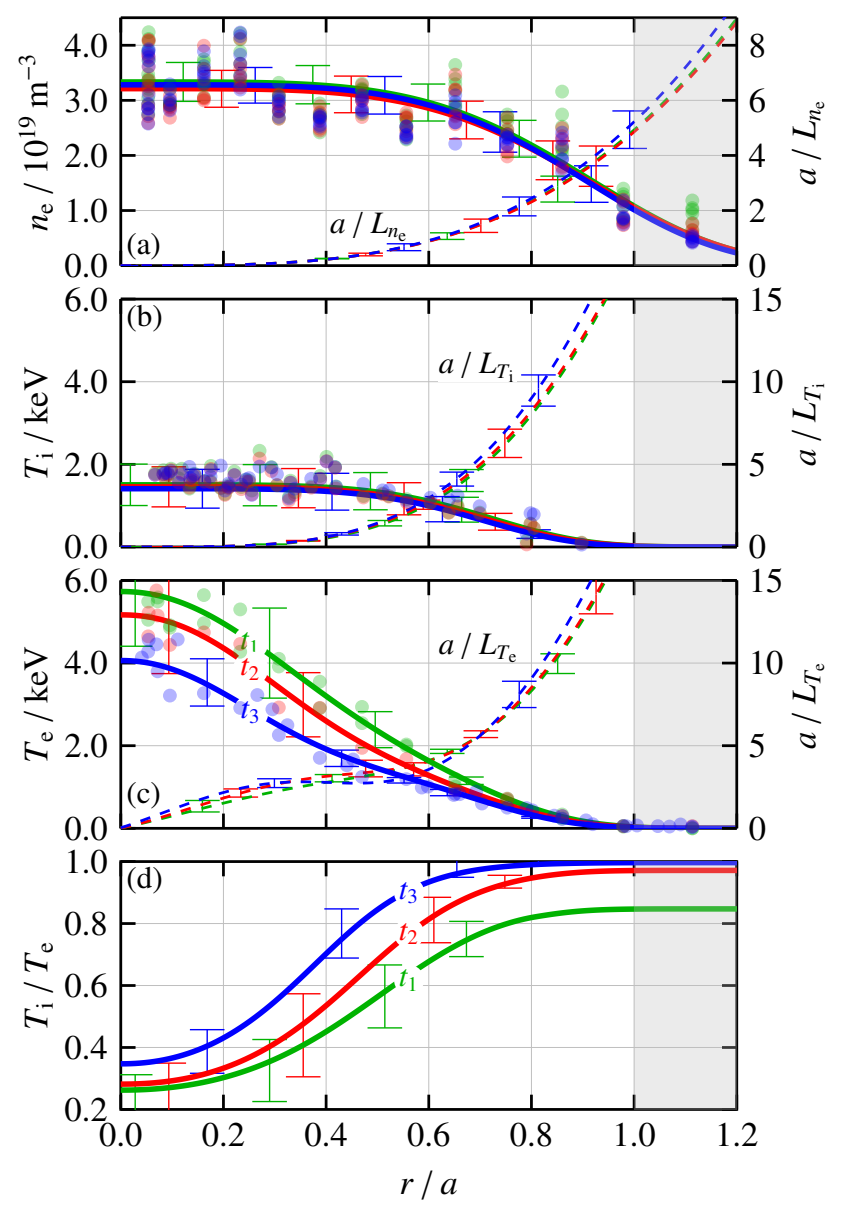

Figure 2. Raw data points $(\bullet)$, bi-parabolic fits (solid lines) and normalized gradient length (dashed lines) of the electron density (a), ion temperature (b), electron temperature (c) and the temperature ratio (d) profiles as a function of the plasma radius at times $t_{1}$ (green), $t_{2}$ (red) and $t_{3}$ (blue) for the experimental program 20180906.038.

troscopy [21] using $100 \mathrm{~ms}$ long neutral beam injection blips. The electron temperature decreases with the ECRH power from $6 \mathrm{keV}$ to $4 \mathrm{keV}$ while the ion temperature and feedback controlled line-integrated electron density [22] remain almost unaffected below $2 \mathrm{keV}$ and at $4 \times 10^{19} \mathrm{~m}^{-2}$, respectively, see figure 1 (b). As shown in figure 1 (a), the plasma stored energy, as analyzed similarly to the previous campaign [23], follows the electron temperature decrease. During each ECRH power step, about $10^{17}$ iron atoms were injected by means of a laser blow-off (LBO) system [24]. These trace amounts of particles barely changed the global plasma parameters but provided intense spectral emission lines of different ionization states of iron. Figure 1 (c) shows the spectroscopic measurement in the ultraviolet [25] and X-ray spectral range [8, 26]. After the LBO injections at times $t_{1}, t_{2}$ and $t_{3}$, the Fe XXV signal exhibits a steep increase, followed by an exponential decay. The decay of the Fe XXV emission provides the impurity transport time $\tau_{\mathrm{I}}$ which increases from about 60 to $90 \mathrm{~ms}$ when reducing the ECRH power. The transport time is a measure for the confinement and transport properties of trace im- 

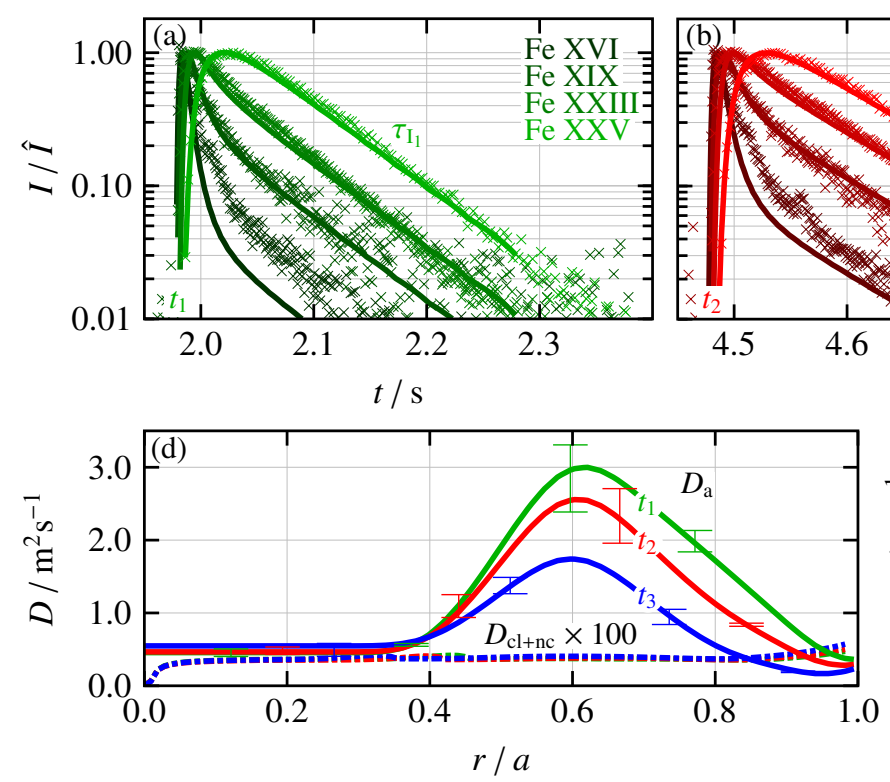
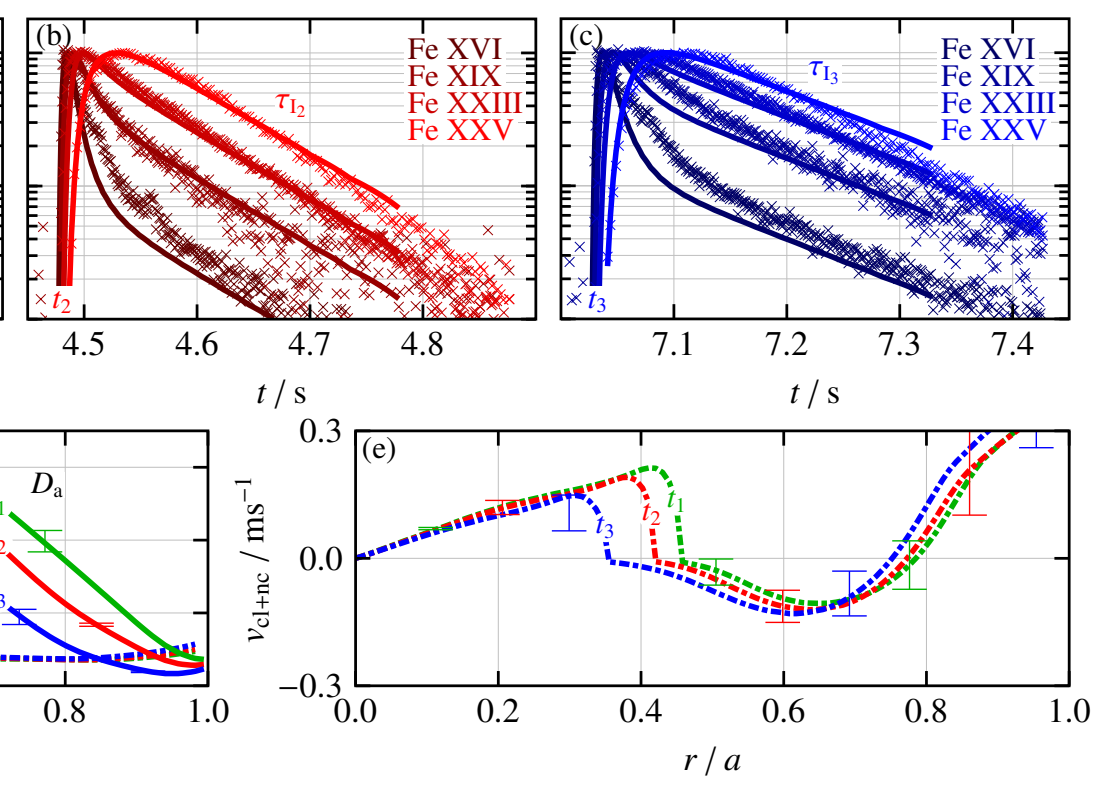

Figure 3. Time traces of emission lines from different iron ionization stages $(\times)$ together with the modeling results (solid line) at times $t_{1}$ (a), $t_{2}$ (b) and $t_{3}$ (c) and the profiles of the anomalous (solid line) and neoclassical (dashed line) diffusion (d) and neoclassical convection (e) coefficients as a function of the plasma radius for the experimental program 20180906.038.

purity ions in the plasma [24]. The intensity of the Fe XXV emission drops with reducing the ECRH power since it scales via the atomic rate coefficients with the electron temperature. Density fluctuation levels were measured by the phase contrast imaging system (PCI) of W7-X [27], which measures poloidally resolved $\left(k_{\vartheta}=k_{\perp}\right)$ fluctuations along a line of sight through the plasma center at a constant toroidal angle, crossing regions of bad curvature where the ITG mode is highly localized in a narrow region [28]. The PCI system is sensitive in the wavenumber-frequency range of both ITG and TEM turbulence. The density fluctuation amplitude was integrated over a frequency range of 20 to $600 \mathrm{kHz}$ and normalized to the line-averaged electron density. As illustrated in figure 1 (d), the amplitude is reduced throughout the different ECRH power steps, indicating a reduced turbulence level, while the impurity transport time increases. It should be noted, that the PCI spectra are uniformly reduced, both in frequency and wavenumber space, demonstrating the broadband nature of the fluctuation.

\section{RESULTS AND DISCUSSION}

The profiles for the three injection times are shown in figure 2. The experimental data (circles) as well as the lower and upper boundary of the $97.5 \%$ probability interval were fitted with a bi-parabolic function (solid line and error bars). As can be seen in figure 2 (a), the density profiles, as well as the normalized density gradient length profiles, are unaltered (within uncertainties) among the three injections. Hence, a possible stabilizing effect of the density gradient should be ruled out. The ion temperature profile as well as the normalized ion temperature gradient length stay the same for the considered timestamps, see figure 2 (b). In contrast, the electron temperature profiles, shown in figure 2 (c), do change in time when decreasing the ECRH power, however the normalized gradient length is not affected. Due to the weak thermal coupling of ions and electrons and the limited ion heat confinement, the core ion temperature is significantly lower compared to the electron temperature. Since all gradient lengths stay essentially the same, we concentrate on the ion to electron temperature ratio, shown in figure 2 (d) for the injection timestamps, which is expected to influence the instability threshold for ITG. As a result of the decreasing electron temperature at constant ion temperatures, the temperature ratio increases. It should be noted, that the uncertainties in the scrape-off layer $(r / a>1)$ are too large, and thus we exclude this region from the analysis.

To investigate the transport properties in more detail, the emission time traces from various charge stages of iron (figure $3 \mathrm{a}$-c) were analyzed with the one-dimensional impurity transport code STRAHL [29]. STRAHL is supplied with the profiles, the neoclassical diffusion and convection velocity profiles from the drift kinetic equation solver (DKES) [30] and infers the anomalous diffusion $D$ and convection $v$ profiles which are needed to match the local impurity flux $\Gamma=-D \nabla n+v n$ as a function of the plasma radius. The iterative optimization procedure of the anomalous diffusion profile is described in detail by Geiger et al. [9]. The results of the least-square fits (solid lines) for the emission from individual ionization stages (crosses) are shown in figure $3(\mathrm{a}-\mathrm{c})$. From figure 3 (d) it is inferred that a two orders of magnitude larger anomalous diffusion coefficient (solid lines) compared to the neoclassical expectations (dotted lines) is required to match the experimental data, as reported also in [9]. Furthermore, the lack of dependence of the transport times on the charge 


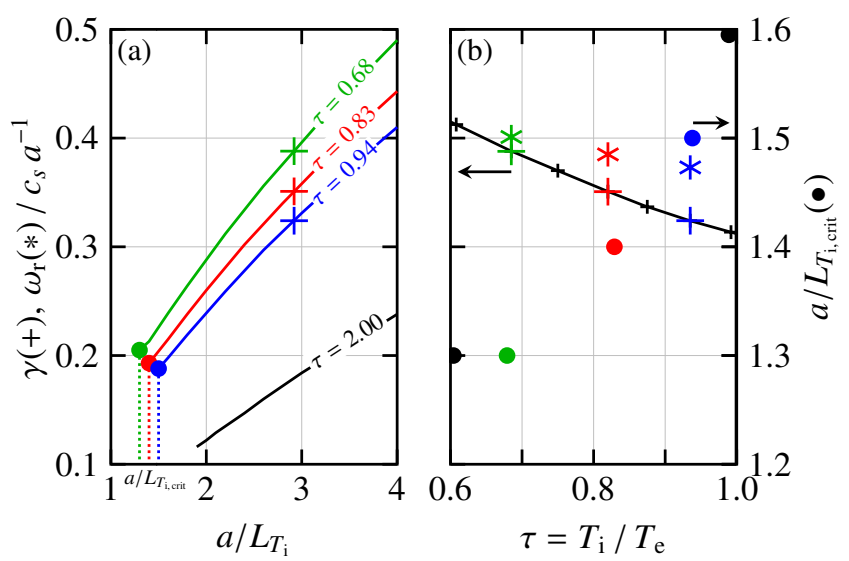

Figure 4. Linear growth rate (solid line and + ) as a function of the normalized ion temperature gradient length for different temperature ratios (a) and over the temperature ratio for $a / L_{T_{\mathrm{i}}}=2.92(\mathrm{~b})$ for the experimental program 20180906.038 at times $t_{1}$ (green), $t_{2}$ (red) and $t_{3}$ (blue). Real frequency of the mode $(*)$ and the critical normalized ion temperature gradient length $(\bullet)$ which is the threshold for the ITG growth rate appearance over the temperature ratio (b).

provides an additional indication that the diffusion of impurities is turbulent $[31,32]$. The low neoclassical diffusion is thought to be the result of the optimization of W7-X with respect to neoclassical transport. The profile of the neoclassical convection velocity does not change dramatically among the three timestamps, see figure 3 (e). The positive neoclassical convection velocity in the center represents a central electronroot confined plasma with outwards directed impurity flux. In the radial region where the anomalous diffusion becomes large, the convection velocity is negative and drives impurities inwards. Ultimately, it is the balanced competition between neoclassical convection and turbulent diffusion that determines the impurity transport.

To study the turbulence mechanism and especially the dependence on the temperature ratio, linear gyro-kinetic simulations with the code GENE in the collisionless electrostatic limit were conducted. Kinetic electrons were considered for these simulations since they are essential in order to capture the fluctuating particle flux. Nevertheless, impurities were not considered as additional species, due to the very small density compared to the bulk plasma. In particular, the effective ion charge of the experimental program considered here is constant for all timestamps at $Z_{\text {eff }}=1.5 \pm 0.4$ and modifies the growth rate by less than $2 \%$, which is well in the range of the uncertainties. A significant change of more than $10 \%$ only appears for $Z_{\text {eff }} \geq 3.5$. The growth rates $\gamma$ as well as the real frequency $\omega_{\mathrm{r}}$ were estimated according to the parameters of this discharge on the surface $r / a=0.6$, where the anomalous diffusion is maximum and the turbulence reaches its strongest level. Gyro-kinetic simulations (not shown here) have established a similar qualitative behavior for other radial locations as well. For each simulation, the maximum linear growth rate was taken in the perpendicular wavenumber spectrum, which remains roughly at the same $k_{\perp} \rho_{s}=0.9$ as the temperature ratio varies. Therefore, a mixing length estimate for the turbulence level, scaling proportional to $\gamma /\left(k_{\perp} \rho_{s}\right)^{2}$, suggests a proportional scaling for the ion heat flux to the growth rate. In figure 4 (a), the growth rates for different normalized temperature gradient lengths $a / L_{T_{\mathrm{i}}}$ were calculated. At the critical normalized ion temperature gradient length $a / L_{T_{\mathrm{i}, \text { crit }}}$, the ITG growth rate starts to appear at ion scales and gets more unstable with $a / L_{T_{\mathrm{i}}}$. As mentioned above, this threshold indeed increases with the temperature ratio, see figure 4 (b), and reduces the growth rate which takes the normalized temperature and density gradient length at $r / a=0.6$ into account. This trend of a depleting turbulence level is consistent with the decreasing impurity transport represented by the anomalous diffusion coefficient which drops with increasing temperature ratio, see figure 3 (d). As discussed above, the assumption of a low TEM turbulence level is reasonable since $a / L_{n_{\mathrm{e}}}$ is not exceptionally large. Typical local nonlinear gyro-kinetic simulations using parameters associated to the presented discharges demonstrate that the main turbulence drive stems from ITG, which is modified by the stabilizing effect of the density gradient, thanks to the optimized magnetic configuration of the W7-X stellarator. Additionally, linear growth rate simulations with GENE that scan a parameter space for different gradient lengths [33] show that the ITG mode dominates and the TEM mode plays a minor role for the here considered gradients. This is additionally supported by the positive real frequency, see figure $4(\mathrm{~b})$, pointing to the direction of the ion propagation. Also, an impact of the plasma beta (plasma pressure normalized to magnetic field) on the equilibrium reconstruction and turbulence can be ruled out due to the low volumeaveraged values of maximum $0.49 \%$. These findings imply that the suppressed turbulence, as corroborated by the observed reduced density fluctuation amplitude, can be mainly assigned to ITG modes that can be correlated with enhanced impurity transport times.

In conclusion, we employed a laser blow-off system to investigate impurity transport in the W7-X stellarator. The observed improved impurity confinement, following an increase of the ion to electron temperature ratio, was attributed to suppressed turbulence driven by the ion temperature gradient, as predicted both by numerical simulations and measured electron density fluctuation amplitudes. This remarkable finding shows that turbulence dominates the transport in W7-X despite the relatively localized region of bad curvature and plays a leading role in the interpretation and prediction of transport in optimized stellarators.

\section{ACKNOWLEDGMENT}

This work has been carried out within the framework of the EUROfusion Consortium and has received funding from the Euratom research and training programme 2014-2018 and 2019-2020 under grant agreement No 633053. The views and opinions expressed herein do not necessarily reflect those of the European Commission. The GENE simulations have been performed on the Marconi (Italy) and the RZG (Germany) supercomputers. The authors thank P. Helander and A. Zocco for helpful discussions. 
[1] H. Maassberg, R. Brakel, R. Burhenn, U. Gasparino, P. Grigull, M. Kick, G. Kühner, H. Ringler, F. Sardei, U. Stroth, and A. Weller, Plasma Physics and Controlled Fusion 35, B319 (1993).

[2] H. Maassberg, C. D. Beidler, and E. E. Simmet, Plasma Physics and Controlled Fusion 41, 1135 (1999).

[3] K. Ida, R. J. Fonck, S. Sesnic, R. A. Hulse, and B. LeBlanc, Phys. Rev. Lett. 58, 116 (1987).

[4] J. Nührenberg and R. Zille, Physics Letters A 114, 129 (1986).

[5] C. Beidler, G. Grieger, F. Herrnegger, E. Harmeyer, J. Kisslinger, W. Lotz, H. Maassberg, P. Merkel, J. Nührenberg, F. Rau, J. Sapper, F. Sardei, R. Scardovelli, A. Schlüter, and H. Wobig, Fusion Technology 17, 148 (1990).

[6] G. Grieger, W. Lotz, P. Merkel, J. Nührenberg, J. Sapper, E. Strumberger, H. Wobig, R. Burhenn, V. Erckmann, U. Gasparino, L. Giannone, H. J. Hartfuss, R. Jaenicke, G. Kühner, H. Ringler, A. Weller, and F. Wagner, Physics of Fluids B: Plasma Physics 4, 2081 (1992).

[7] C. D. Beidler et al., "Successful reduction of neoclassical energy transport in the Wendelstein 7-X stellarator," submitted to Nature.

[8] A. Langenberg, N. Pablant, O. Marchuk, D. Zhang, J. Alonso, R. Burhenn, J. Svensson, P. Valson, D. Gates, M. Beurskens, R. Wolf, and the W7-X Team, Nuclear Fusion 57, 086013 (2017).

[9] B. Geiger, Th. Wegner, C. Beidler, R. Burhenn, B. Buttenschön, R. Dux, A. Langenberg, N. A. Pablant, T. Pütterich, Y. Turkin, T. Windisch, V. Winters, M. Beurskens, C. Biedermann, K. J. Brunner, G. Cseh, H. Damm, F. Effenberg, G. Fuchert, O. Grulke, J. Harris, C. Killer, J. Knauer, G. Kocsis, A. KrämerFlecken, T. Kremeyer, M. Krychowiak, O. Marchuk, D. Nicolai, K. Rahbarnia, G. Satheeswaran, J. Schilling, O. Schmitz, T. Schröder, T. Szepesi, H. Thomsen, H. Trimiño Mora, P. Traverso, D. Zhang, and the W7-X Team, Nuclear Fusion 59, 046009 (2019).

[10] F. Jenko, W. Dorland, M. Kotschenreuther, and B. N. Rogers, Physics of Plasmas 7, 1904 (2000).

[11] G. G. Plunk, P. Xanthopoulos, G. M. Weir, S. A. Bozhenkov, A. Dinklage, G. Fuchert, J. Geiger, M. Hirsch, U. Höfel, M. Jakubowski, A. Langenberg, N. Pablant, E. Pasch, T. Stange, D. Zhang, and the W7-X Team, Phys. Rev. Lett. 122, 035002 (2019).

[12] J. H. E. Proll, P. Helander, J. W. Connor, and G. G. Plunk, Phys. Rev. Lett. 108, 245002 (2012).

[13] J. H. E. Proll, P. Xanthopoulos, and P. Helander, Physics of Plasmas 20, 122506 (2013).

[14] W. Horton, D. Choi, and W. M. Tang, The Physics of Fluids 24, 1077 (1981).

[15] F. Romanelli, Physics of Fluids B: Plasma Physics 1, 1018 (1989).

[16] X. Q. Xu and M. N. Rosenbluth, Physics of Fluids B: Plasma Physics 3, 627 (1991).

[17] A. Casati, C. Bourdelle, X. Garbet, and F. Imbeaux, Physics of Plasmas 15, 042310 (2008).
[18] A. Zocco, P. Xanthopoulos, H. Doerk, J. W. Connor, and P. Helander, Journal of Plasma Physics 84, 715840101 (2018).

[19] T. Andreeva, Vacuum Magnetic Configurations of Wendelstein 7-X, IPP III/270 (Garching: Max-Planck-Institut für Plasmaphysik, 2002).

[20] E. Pasch, M. N. A. Beurskens, S. A. Bozhenkov, G. Fuchert, J. Knauer, and R. C. Wolf, Review of Scientific Instruments 87, 11E729 (2016).

[21] O. P. Ford, L. Vanó, J. A. Alonso, J. Baldzuhn, M. N. A. Beurskens, C. Biedermann, S. A. Bozhenkov, G. Fuchert, B. Geiger, D. Hartmann, R. J. E. Jaspers, A. Kappatou, A. Langenberg, S. A. Lazerson, R. M. McDermott, P. McNeely, T. W. C. Neelis, N. A. Pablant, E. Pasch, N. Rust, R. Schroeder, E. R. Scott, H. M. Smith, Th. Wegner, F. Kunkel, R. C. Wolf, and the W7-X Team, Review of Scientific Instruments 91, 023507 (2020).

[22] K. J. Brunner, T. Akiyama, M. Hirsch, J. Knauer, P. Kornejew, B. Kursinski, H. Laqua, J. Meineke, H. Trimiño Mora, and R. C. Wolf, Journal of Instrumentation 13, P09002 (2018).

[23] K. Rahbarnia, H. Thomsen, U. Neuner, J. Schilling, J. Geiger, G. Fuchert, T. Andreeva, M. Endler, D. Hathiramani, T. Bluhm, M. Zilker, B. Carvalho, A. Werner, and the W7-X Team, Nuclear Fusion 58, 096010 (2018).

[24] Th. Wegner, B. Geiger, F. Kunkel, R. Burhenn, T. Schröder, C. Biedermann, B. Buttenschön, G. Cseh, P. Drews, O. Grulke, K. Hollfeld, C. Killer, G. Kocsis, T. Krings, A. Langenberg, O. Marchuk, U. Neuner, D. Nicolai, G. Offermanns, N. A. Pablant, K. Rahbarnia, G. Satheeswaran, J. Schilling, B. Schweer, T. Szepesi, H. Thomsen, and the W7-X Team, Review of Scientific Instruments 89, 073505 (2018).

[25] W. Biel, G. Bertschinger, R. Burhenn, R. König, and E. Jourdain, Review of Scientific Instruments 75, 3268 (2004).

[26] G. Bertschinger, W. Biel, H. Jaegers, and O. Marchuk, Review of Scientific Instruments 75, 3727 (2004).

[27] E. M. Edlund, M. Porkolab, Z. Huang, O. Grulke, L.-G. Böttger, C. von Sehren, and A. von Stechow, Review of Scientific Instruments 89, 10E105 (2018).

[28] A. Bañón Navarro et al., "Global gyrokinetic simulations of ITG turbulence in the magnetic configuration space of the Wendelstein 7-X stellarator," submitted to Plasma Physics and Controlled Fusion.

[29] K. Behringer, Description of the Impurity Transport Code "STRAHL", JET-R(87) 08 (JET Joint Undertaking, Abingdon, Oxfordshire, 1987).

[30] S. P. Hirshman, K. C. Shaing, W. I. van Rij, C. O. Beasley, and E. C. Crume, The Physics of Fluids 29, 2951 (1986).

[31] P. Helander and A. Zocco, Plasma Physics and Controlled Fusion 60, 084006 (2018).

[32] A. Langenberg, Th. Wegner, N. A. Pablant, O. Marchuk, B. Geiger, N. Tamura, R. Bussiahn, M. Kubkowska, A. Mollén, P. Traverso, H. M. Smith, G. Fuchert, S. Bozhenkov, H. Damm, E. Pasch, K.-J. Brunner, J. Knauer, M. Beurskens, R. Burhenn, R. C. Wolf, and the W7-X Team, Physics of Plasmas 27, 052510 (2020).

[33] J. A. Alcusón, P. Xanthopoulos, G. G. Plunk, P. Helander, F. Wilms, Y. Turkin, A. von Stechow, and O. Grulke, Plasma Physics and Controlled Fusion 62, 035005 (2020). 